\title{
PT-symmetric eigenvalues for homogeneous potentials
}

\author{
Alexandre Eremenko* and Andrei Gabrielov
}

November 21, 2017

\begin{abstract}
We consider one-dimensional Schrödinger equations with potential $x^{2 M}(i x)^{\varepsilon}$, where $M \geq 1$ is an integer, and $\varepsilon$ is real, under appropriate PT-symmetric boundary conditions. We prove the phenomenon which was discovered by Bender and Boettcher by numerical computation: as $\varepsilon$ changes, the real spectrum suddenly becomes non-real in the sense that all but finitely many eigenvalues become non-real. We find the limit arguments of these non-real eigenvalues $E$ as $E \rightarrow \infty$.
\end{abstract}

MSC: 81Q05, 34M60, 34A05.

Keywords: one-dimensional Schrödinger operators, PT-symmetry, WKB, asymptotic expansions, entire functions.

\section{Introduction.}

Anharmonic oscillators (one-dimensional Schrödinger operators with polynomial potentials) played an important role in quantum mechanics since its inception [14], 5], 44, [20]. In the early 1990s, Bessis and Zin-Justin made a surprising conjecture that the eigenvalue problem

$$
-w^{\prime \prime}+i x^{3} w=E w, \quad w( \pm \infty)=0,
$$

has real discrete spectrum. Their original work is not published but the history and motivation are described in the survey [9]. Stimulated by this conjecture, Bender and Boettcher [1], [2] proposed to study the one-parametric

*Both authors are supported by NSF grant DMS-1665115. 
families of equations with homogeneous potentials which contain Airy's equation, harmonic oscillators, and anharmonic oscillators with cubic and quartic homogeneous potentials. This work of Bender and Boettcher started a new large area of research which is called PT-symmetric quantum mechanics. PT stands for "parity and time", and in our context this means that the potential $V$ of the operator

$$
-\frac{d^{2}}{d x^{2}}+V(x)
$$

satisfies $V(-\bar{x})=\overline{V(x)}$ and the boundary conditions are interchanged by the transformation $x \mapsto-\bar{x}$.

Bender and Boettcher considered the equation

$$
-w^{\prime \prime}+x^{2 M}(i x)^{\varepsilon} w=E w,
$$

with a positive integer $M$ and real $\varepsilon$. Here the principal branch of the power is used, so the branch cut in (2) is the positive imaginary ray. The boundary condition for $\varepsilon=0$ is imposed on the real line: $w( \pm \infty)=0$, so that for $\varepsilon=0$ we obtain the harmonic oscillator when $M=1$, and a quartic oscillator when $M=2$. When $|\varepsilon|$ increases and becomes large, the boundary condition has to be continuously deformed (to preserve the discrete spectrum), so that $w(z) \rightarrow 0$ on two normalization rays in the complex plane which lay inside non-adjacent Stokes sectors symmetric with respect to the imaginary line.

We recall that Stokes sectors are the sectors between Stokes lines, and Stokes lines are defined by $V(z) d z^{2}<0$, where $V$ is the potential in (11). In the case of a homogeneous potential in (2), Stokes lines are equally spaced rays with angles $2 \pi /(m+2)$ between adjacent rays, where

$$
m=2 M+\epsilon .
$$

The negative imaginary ray is a Stokes ray when $M$ is even, and bisects a Stokes sector when $M$ is odd. Rotation of a normalization ray within its Stokes sector does not change the normalization. So the normalization conditions just tell us in which pair of Stokes sectors eigenfunctions tend to zero.

It is convenient to define the level of the PT-symmetric problem as the number of full Stokes sectors between the normalization rays. Thus the level equals $M \geq 1$ in (2) for the problem considered by Bender and Boettcher.

Such eigenvalue problems, with normalization on two rays in the complex plane, were studied for the first time by Sibuya [18] for complex polynomial potentials, without any symmetry conditions. 


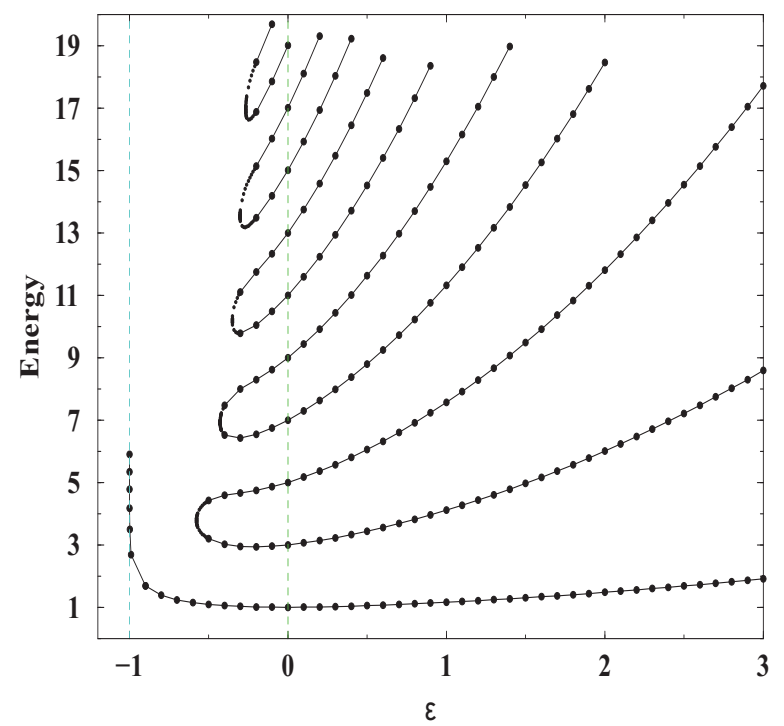

Figure 1: Real eigenvalues of $-w^{\prime \prime}+x^{2}(i x)^{\varepsilon} w$ as functions of $\varepsilon$ ([2, Fig. 11]).

Eigenvalues of any PT symmetric problem are symmetric with respect to the real line. Bender and Boettcher were interested in reality of eigenvalues, and they obtained by numerical computation the following remarkable figures: Fig. 1 (level 1), Fig. 2 (level 2) and Fig. 3 (level 3) which we reproduced from [2].

The most conspicuous feature of these figures is a "phase transition": for $\varepsilon>0$ all eigenvalues are real, while for $\varepsilon<0$ they are all real when $\varepsilon$ is an integer (such that $\varepsilon+2 M>1$ ), while almost all of them are non-real for negative non-integer $\varepsilon$. Here and in what follows "almost all" means "all but finitely many".

The purpose of this paper is to prove these facts. We do this for $M=1$ and $M=2$, but it seems that our method can be extended to arbitrary integer $M \geq 1$.

Now we state some known rigorous results about reality of eigenvalues of (2). In [16], K. Shin proved that that for any potential $x^{2 M}(i x)^{\varepsilon}$ with integers $M, \varepsilon, 2 M+\varepsilon>1$, and for arbitrary PT-symmetric boundary conditions at infinity, all eigenvalues are real.

Shin's proof consists of two parts: $\varepsilon \geq 0$ and $\varepsilon<0$. The part about $\varepsilon \geq 0$ works also for non-integer $\varepsilon$, see also [11] for $M=1$ and $M=2$. In the case $\varepsilon<0$, Shin makes the change of the variable $x \mapsto-x$ to reduce to the case 


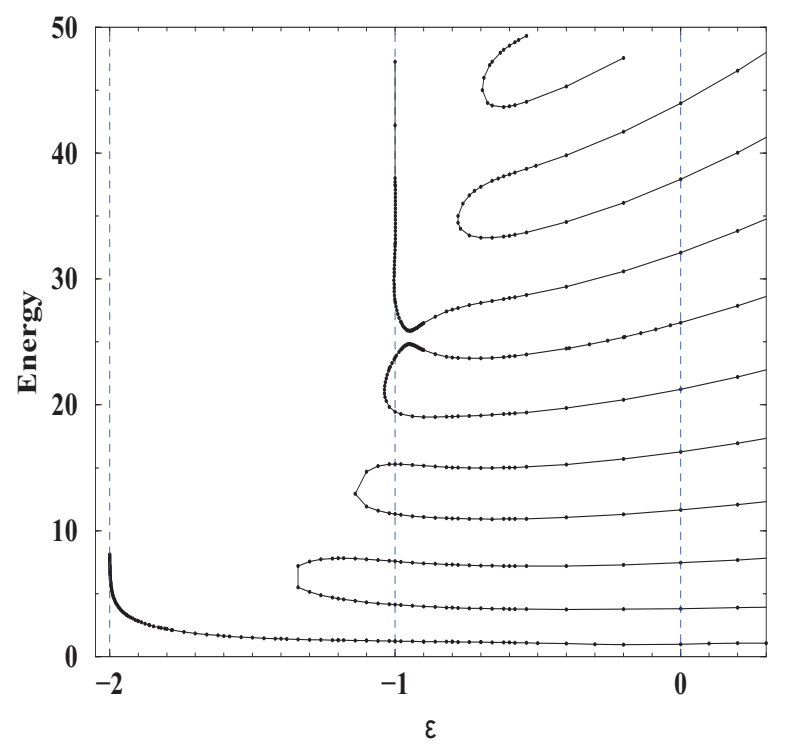

Figure 2: Real eigenvalues of $-w^{\prime \prime}+x^{4}(i x)^{\varepsilon} w$ as functions of $\varepsilon$. The almost vertical part over $\varepsilon=-1$ indicates that all eigenvalues are real: it actually wiggles about the vertical line $\varepsilon=-1$ ([2, Fig. 14]).

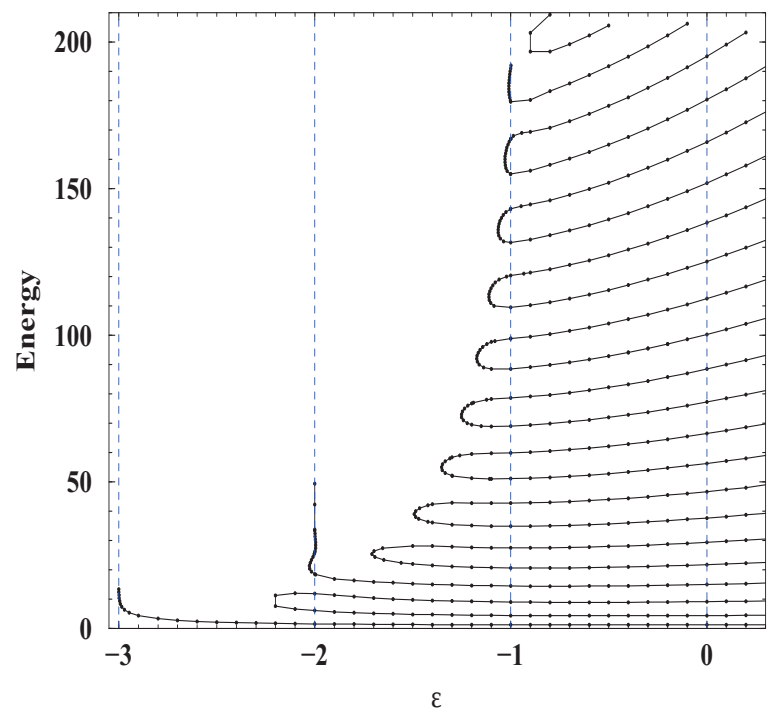

Figure 3: Real eigenvalues of $-w^{\prime \prime}+x^{6}(i x)^{\varepsilon} w$ as functions of $\varepsilon$. All eigenvalues are real for $\varepsilon=-1$ and $\varepsilon=-2$. For non-integer $\varepsilon<0$ almost all of them are non-real ([2, Fig. 20]). 
$\epsilon>0$, which of course can be done only when $\varepsilon$ is an integer. In fact, the figures of Bender and Boettcher and our theorems stated below show that for negative non-integer $\epsilon$ almost all eigenvalues are non-real.

All reality proofs are based on the method invented by Dorey, Dunning and Tateo, who proved the original conjecture of Bessis and Zinn-Justin in [7. These authors discovered that spectral determinants (entire functions whose zeros are eigenvalues) arising in PT-symmetric eigenvalue problems that we consider here arise also in integrable models of statistical mechanics on 2-dimensional lattices. This gives an additional reason to study these entire functions. A comprehensive survey of their results on this ODE/IM correspondence is given in [8].

The case of non-integer $\varepsilon<0$ was considered in [10], but in that paper the authors only study the first few eigenvalues, and small values of $\varepsilon$.

Our results are the following.

Theorem 1. For the equation (圆) with $M=1, \varepsilon \in(-1,0)$ and the boundary conditions $w(x) \rightarrow 0, x \rightarrow \pm \infty$, almost all eigenvalues are non-real. The arguments of the eigenvalues accumulate to

$$
\pm \pi \frac{2-m}{2+m}, \quad \text { as } \quad E \rightarrow \infty
$$

where $m=2 M+\epsilon$.

Theorem 2. For the equation (圆) with $M=2, \varepsilon \in(-3,0) \backslash\{-1,-2\}$ and the boundary conditions

$$
w(x) \rightarrow 0, \quad x \rightarrow \infty, \quad \arg x=-\frac{\pi}{2} \pm \frac{3 \pi}{m+2},
$$

almost all eigenvalues are non-real, and their arguments accumulate to

$$
\pm \pi \frac{4-m}{2+m}, \quad \text { as } \quad E \rightarrow \infty
$$

where $m=2 M+\epsilon$.

When $\epsilon \in(-2,0)$ the boundary condition (4) is equivalent to $w(x) \rightarrow$ $0, x \rightarrow \pm \infty$.

Our method is different from that of [10]. The plan is the following. First we introduce an auxiliary self-adjoint problem with real discrete spectrum, and an entire function $f$ which is called a spectral determinant whose zeros 
are exactly the eigenvalues of this self-adjoint problem. Improving the previous results of Sibuya and Shin, we obtain an asymptotic expansion of this spectral determinant $f$. Then we use the important fact that the spectral determinants of the PT-symmetric problems are also entire functions and that they have explicit expressions in terms of $f$, see [19, 8, 16, 11]. By studying the asymptotic behavior of these spectral determinants we can make conclusions about the asymptotic distribution of their zeros as $E \rightarrow \infty$. This permits us to find their limit arguments. To show that there are only finitely many real eigenvalues, we combine the asymptotics with the "cancellation conditions" in the formulas for the spectral determinants.

In [2] and [10] doubts are expressed about applicability of the WKB method for non-integer exponent (3), especially when $m<2$. In fact the proof of our main technical result, Theorem 3, can be considered as a refinement of the usual WKB method, adapted for handling non-integer $m$.

When $M=1, \varepsilon \rightarrow-1$ and when $M=2, \varepsilon \rightarrow-2$, the Stokes sectors containing the normalization rays became adjacent and the limit problem (for the Airy equation in the first case and for the quadratic potential in the second) cannot have eigenvalues, so all eigenvalues escape to infinity.

Numerical evidence of what happens when $\varepsilon$ is below these lower limits is presented in the recent paper [3]. The method of our paper breaks down when $m=2 M+\varepsilon \leq 1$.

\section{Auxiliary self-adjoint problem.}

Consider the differential equation

$$
y^{\prime \prime}=\left(z^{m}+\lambda\right) y, \quad \text { where } \quad m>1, m \neq 2 .
$$

All powers and logarithms are understood in the sense of principal branches: $|\arg z|<\pi$, unless some other branch is explicitly specified.

Sibuya's theorem. For every $\lambda \in \mathbf{C}$ there exists a unique solution $y_{0}(z, \lambda)$ on the positive ray such that

$y_{0}(z, \lambda)=(1+o(1)) z^{-m / 4} \exp \left(-\frac{2}{m+2} z^{(m+2) / 2}-\frac{\lambda}{2-m} z^{(2-m) / 2}\right), \quad z \rightarrow+\infty$.

This solution has an analytic continuation in $z$ to $\mathbf{C} \backslash(-\infty, 0]$, and the asymptotic formula (6) holds in every sector

$$
\{z:|\arg z| \leq 3 \pi /(m+2)-\epsilon\}, \quad \epsilon>0 .
$$


When $m>2$, the second term under the exponent in (6) is redundant.

The limits

$$
f(\lambda):=\lim _{z \rightarrow 0+} y_{0}(z, \lambda), \quad \text { and } \quad f_{1}(\lambda):=\lim _{z \rightarrow 0+} y_{0}^{\prime}(z, \lambda)
$$

exist, and both are entire functions of $\lambda$, of order

$$
\rho=\frac{1}{2}+\frac{1}{m} \text {. }
$$

Comments. Sibuya stated this theorem for integer $m$, but his proof in [18, Ch. 2] requires only minor modification to deal with real $m>1$. In [8, 10, 19], Sibuya's theorem is used for non-integer $m$. We now explain the necessary modifications.

As the first step, one changes the independent variable to $\zeta=\sqrt{z}$ and by some changes of the dependent variables reduces (5) to the following Riccati equation:

$$
\begin{aligned}
\zeta^{-m-1} p^{\prime}(\zeta) & =\left(\lambda \zeta^{-2 m}+(m / 2) \zeta^{-2-m}\right) p^{2}+\left(4+2 \lambda \zeta^{-2 m}\right) p \\
& +\lambda \zeta^{-2 m}-(m / 2) \zeta^{-2-m}
\end{aligned}
$$

Then one shows [18, Lemma 9.1] that this Riccati equation has a formal solution of the form

$$
p(\zeta)=\sum_{n=1}^{\infty} p_{n} \zeta^{-n}
$$

This is where the difference between integer and non-integer $m$ arises. When $m$ is not an integer, one considers the additive semi-group

$$
\Lambda=\left\{k_{1}+k_{2} m: k_{1}, k_{2} \in \mathbf{Z}_{\geq 0}\right\}
$$

and finds a unique formal solution of the Riccati equation in the form

$$
p(\zeta)=\sum_{\beta \in \Lambda} p_{\beta} \zeta^{-\beta} .
$$

It is easy to see that such a formal solution exists and is unique. Then one checks that the basic results on the asymptotic expansions (theorems 3.3, 4.1 and 5.1 in [18]) extend without changes to the asymptotic expansions of the form (9). For such general asymptotic expansions, see, for example, [6]. 
The concluding step, showing that there is a true solution corresponding to the asymptotic expansion (91), and this solution is an entire function of $\lambda$, is achieved by reducing the Riccati equation to an integral equation and solving this integral equation by iteration. This last step requires no changes for non-integer $m$.

Sibuya [18, Thm. 19.1] also studied the asymptotics of $f$ and $f_{1}$, and found the principal terms

$$
\begin{gathered}
f(\lambda)=(1+o(1)) \lambda^{-1 / 4} \exp \left(K_{m} \lambda^{\rho}\right), \\
f_{1}(\lambda)=-(1+o(1)) \lambda^{1 / 4} \exp \left(K_{m} \lambda^{\rho}\right),
\end{gathered}
$$

for $m>2$. Here $K_{m}>0$ is an explicit constant. The error term estimates were improved by Shin [17] who proved

$$
\begin{gathered}
f(\lambda)=\left(1+O\left(\lambda^{-\rho}\right)\right) \lambda^{-1 / 4} \exp \left(K_{m} \lambda^{\rho}\right), \\
f_{1}(\lambda)=-\left(1+O\left(\lambda^{-\rho}\right)\right) \lambda^{1 / 4} \exp \left(K_{m} \lambda^{\rho}\right) .
\end{gathered}
$$

These asymptotics hold when $|\lambda| \rightarrow \infty$, uniformly in every sector

$$
\{\lambda:|\lambda| \leq \pi-\epsilon\} .
$$

They can be also extended to a sector containing the negative ray in the following way:

$$
\begin{aligned}
f(\lambda) & =\left(1+O\left(\lambda^{-\rho}\right)\right) \lambda^{-1 / 4} \exp \left(K_{m} \lambda^{\rho}\right) \\
& +\left(i+O\left(\lambda^{-\rho}\right)\right) \lambda^{-1 / 4} \exp \left(e^{-2 \pi i \rho} K_{m} \lambda^{\rho}\right)
\end{aligned}
$$

as $\lambda \rightarrow \infty, \pi-\epsilon<\arg \lambda<\pi+\epsilon$, with some $\epsilon>0$. Similar result holds for $f_{1}$. Asymptotics near the negative ray permitted Shin [17, Cor. 2.1] to locate the zeros of $f$ with the error smaller than the distance to the nearest other zero. These zeros are negative (see below). All these results of Sibuya and Shin generalize without changes to the case of non-integer $m>1$, except that $K_{m}<0$ for $m \in(1,2)$.

We will need the following more precise asymptotics of $f$.

Theorem 3. Suppose that $m>1$, and $m$ is not an integer. Function $f$ in Sibuya's theorem has all zeros negative, and the following asymptotics holds

$$
f(\lambda)=\lambda^{-1 / 4} \Psi\left(\lambda^{\rho}\right) \exp \left(K_{m} \lambda^{\rho}\right)
$$


where

$$
\Psi(\mu)=\left(1+\sum_{n=1}^{[m]} c_{n} \mu^{-n}+(1 / 8) \Gamma(m+1) \mu^{-m}+O\left(\mu^{-\kappa}\right)\right),
$$

as $\mu=\lambda^{\rho}$, and $|\lambda| \rightarrow \infty$ in every sector (12). Here $\kappa>m$.

Explicit expressions for $K_{m}, c_{1}$ will be given later, they are irrelevant for our main argument, except the sign of $K_{m}$. Presence of the term $\lambda^{-m \rho}$ in the asymptotics is crucial.

Proof. First we show that all zeros of $f$ are negative. Indeed, if $f(\lambda)=0$ then $-\lambda$ is an eigenvalue of the Sturm-Liouville problem on the positive ray:

$$
-y^{\prime \prime}+z^{m} y=-\lambda y, \quad y(0)=y(+\infty)=0,
$$

with positive potential, so $-\lambda>0$.

In the equation (5), we make the change of the variable

$$
w(z)=y\left(\lambda^{1 / m} z\right), \quad y(z)=w\left(\lambda^{-1 / m} z\right),
$$

and obtain

$$
w^{\prime \prime}=\mu^{2}\left(z^{m}+1\right) w, \quad \text { where } \quad \mu:=\lambda^{\rho},
$$

where $\rho$ is defined in (7). So we consider the differential equation

$$
w^{\prime \prime}=\mu^{2} Q(z) w, \quad \text { where } \quad Q(z)=z^{m}+1 .
$$

Following Liouville, we introduce the new independent variable

$$
\zeta=\Phi(z)=\int_{0}^{z} \sqrt{Q(t)} d t .
$$

This function is well defined in the sector $|\arg z|<\pi / m$, and maps this sector onto the region $\Omega_{m}$ in the $\zeta$-plane (see Fig. 4), which contains the positive ray and is symmetric with respect to the real line. If $m \in(1,2)$ this region $\Omega_{m}$ overlaps itself, and has to be considered as a Riemann surface spread over the $\zeta$-plane.

On the real line $\Phi$ is strictly increasing, $\Phi^{\prime}(0)=1$ and $\Phi(+\infty)=+\infty$. Notice that the region $\Omega_{m}$ contains the sector $|\arg \zeta|<\pi / m$.

Function $\Phi$ has a convergent expansion near 0 with exponents of the set $\Lambda$ as in (8) :

$$
\Phi(z)=z+\frac{1}{2(m+1)} z^{m+1}+\ldots
$$




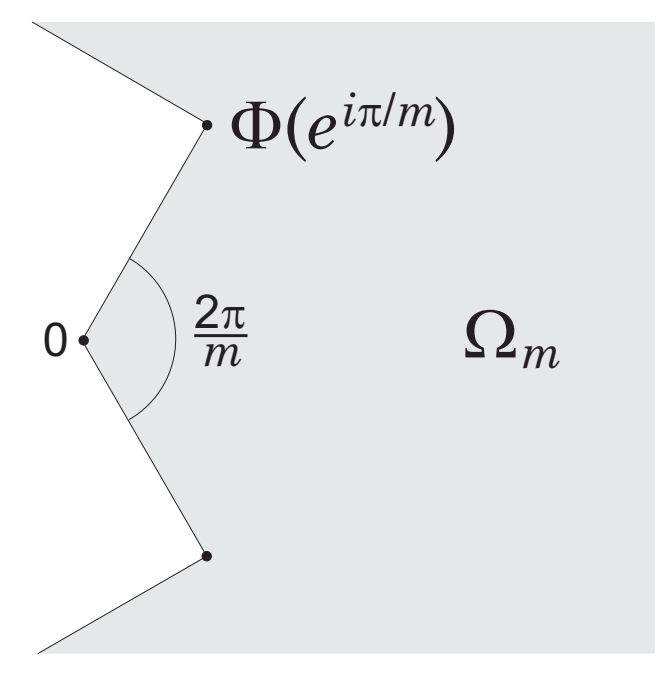

Figure 4: The region $\Omega_{m}$ for $m=3$.

The asymptotics of $\Phi$ at $\infty$ is

$$
\Phi(z) \sim \frac{1}{m+1} z^{(m+2) / 2}, \quad z \rightarrow \infty .
$$

We introduce a new function $u(\zeta, \mu)$ in $\Omega_{m}$ :

$$
u(\zeta, \mu)=Q^{1 / 4}(z) w(z), \quad \text { where } \quad z=\Phi^{-1}(\zeta) .
$$

According to Liouville, see for example [12], this function satisfies

$$
u^{\prime \prime}=\mu^{2} u+g u, \quad \text { where } \quad u^{\prime \prime}=d^{2} u / d \zeta^{2},
$$

and

$$
g(\zeta)=\left(-\frac{5}{16} \frac{Q^{\prime 2}}{Q^{3}}+\frac{Q^{\prime \prime}}{4 Q^{2}}\right) \circ \Phi^{-1}(\zeta)
$$

Notice that $g$ is integrable on every ray in $\Omega_{m}$, in fact a simple calculation using (22) shows that

$$
g(\zeta)=O\left(\zeta^{-2}\right), \quad \zeta \rightarrow \infty, \quad \zeta \in \Omega_{m}
$$

Near the origin, $g$ has a convergent expansion with exponents in $\Lambda-2$ :

$$
g(\zeta)=\frac{1}{4} m(m-1) \zeta^{m-2}+\ldots
$$


Considering $g u$ as the right hand side of the non-homogeneous linear differential equation (24) and using the variation of constants formula, we obtain an integral equation for $u$ :

$$
u(\zeta, \mu)=e^{-\mu \zeta}+\frac{1}{\mu} \int_{\zeta}^{\infty} \sinh \mu(t-\zeta) g(t) u(t, \mu) d t .
$$

Here $\zeta \in \Omega_{m}$ and the integration is along any path in $\Omega_{m}$ from $\zeta$ to $\infty$.

It is easy to check directly that a solution of this integral equation, if exists, satisfies (24). We simplify our integral equation by introducing the function

$$
F(\zeta, \mu)=e^{\mu \zeta} u(\zeta, \mu)
$$

Then

$$
F(\zeta, \mu)=1+\frac{1}{2 \mu} \int_{\zeta}^{\infty}\left(1-e^{\mu(\zeta-t)}\right) g(t) F(t, \mu) d t
$$

Suppose first that $\lambda, \mu$ and $\zeta$ are positive, and the integration in (30) is along the positive ray. We will construct a solution $F$ of this integral equation in $\Omega_{m}$ which tends to 1 as $\zeta \rightarrow+\infty$. Then we will set according to our changes of the variables

$$
y_{0}(z, \lambda)=c(\lambda) Q^{-1 / 4}\left(\lambda^{-1 / m} z\right) \exp \left(-\lambda^{\rho} \Phi\left(\lambda^{-1 / m} z\right)\right) F\left(\Phi^{-1}\left(\lambda^{-1 / m} z\right), \lambda^{\rho}\right),
$$

where

$$
c(\lambda)=\lambda^{-1 / 4} e^{K_{m} \lambda^{\rho}}
$$

and

$$
\begin{gathered}
K_{m}=\int_{0}^{\infty}\left(\sqrt{t^{m}+1}-t^{m / 2}\right) d t>0, \quad \text { when } m>2, \\
K_{m}=\int_{0}^{\infty}\left(\sqrt{t^{m}+1}-t^{m / 2}-(1 / 2) t^{-m / 2}\right) d t<0, \quad \text { when } \quad 1<m<2 .
\end{gathered}
$$

It is easy to verify using (29), (23), (17) and (20), that this solution $y_{0}$ has the correct asymptotics (6) as $z \rightarrow+\infty$. Expression (31) is the WKB solution of (5),

$$
y_{0}(z, \lambda)=\left(c_{1}(\lambda)+o(1)\right)\left(z^{m}+\lambda\right)^{-1 / 4} \exp \left(-\int_{0}^{z} \sqrt{t^{m}+\lambda} d t\right),
$$

where the constant factor $c_{1}(\lambda)=e^{K_{m} \lambda^{\rho}}$ is chosen so that the dependence of the exponential term in the asymptotics ([6) on $\lambda$ is as simple as possible: 
when $m>2$ it does not depend on $\lambda$ at all, while for $m \in(1,2)$ it is a simple entire function of $\lambda$.

It is easy to verify directly that $y_{0}$ defined by (31) formally satisfies (5) if $F$ satisfies (30).

Existence of a solution of (30). We set $F_{0}=0, F_{n}=\mathbf{I}_{\mu}\left(F_{n-1}\right)$, where $\mathbf{I}_{\mu}$ is the integral operator in the right hand side of (30). We assume first that $\mu>0, \zeta>0$ and that integration is over the positive ray. Since

$$
\left|F_{n}-F_{n+1}\right|(\zeta) \leq \frac{1}{2 \mu} \int_{0}^{\infty}|g(t)|\left|F_{n}(t)-F_{n-1}(t)\right| d t \leq \frac{1}{2 \mu}\|g\|_{1}\left\|F_{n}-F_{n-1}\right\|_{\infty},
$$

the series

$$
F=F_{1}+\left(F_{2}-F_{1}\right)+\left(F_{3}-F_{2}\right)+\ldots
$$

converges uniformly on $[0, \infty)$ when $\mu>\mu_{0}$, and defines a bounded function $F$ solving (30). We also have $|F-1| \leq$ const $/ \mu$.

Asymptotic expansion of $F(0, \mu)$ for $\mu>0$. Equation (30) is of the form

$$
F=\mathbf{1}+\frac{1}{2 \mu} \mathbf{T}_{\mu}[F]
$$

where $\mathbf{T}_{\mu}=2 \mu\left(\mathbf{I}_{\mu}-\mathbf{1}\right)$ is the integral operator

$$
\mathbf{T}_{\mu}[F](\zeta)=\int_{\zeta}^{\infty}\left(1-e^{\mu(\zeta-t)}\right) g(t) F(t) d t
$$

This equation is satisfied by the formal series

$$
F=\mathbf{1}+\sum_{n=1}^{\infty}(2 \mu)^{-n} \mathbf{T}_{\mu}^{n}[\mathbf{1}]
$$

We set $\zeta=0$ in this series, and obtain

$$
\begin{gathered}
\mathbf{T}_{\mu}[\mathbf{1}](0)=\int_{0}^{\infty}\left(1-e^{-\mu t}\right) g(t) d t \\
\mathbf{T}_{\mu}^{2}[\mathbf{1}](0)=\int_{0}^{\infty}\left(1-e^{-t \mu}\right) g(t) \int_{t}^{\infty}\left(1-e^{\mu\left(t-t_{1}\right)}\right) g\left(t_{1}\right) d t_{1} d t
\end{gathered}
$$

and so on,

$$
\begin{aligned}
\mathbf{T}_{\mu}^{n}[\mathbf{1}](0) & =\int_{0}^{\infty}\left(1-e^{-\mu t}\right) g(t) \int_{t}^{\infty}\left(1-e^{\mu\left(t-t_{1}\right)}\right) g\left(t_{1}\right) \\
& \times \int_{t_{1}}^{\infty}\left(1-e^{\mu\left(t_{1}-t_{2}\right)}\right) g\left(t_{2}\right) \ldots \\
& \times \int_{t_{n-2}}^{\infty}\left(1-e^{\mu\left(t_{n-2}-t_{n-1}\right)}\right) g\left(t_{n-1}\right) d t_{n-1} d t_{n-2} \ldots d t_{1} d t
\end{aligned}
$$


The $n$-th term of the series (33) is $O\left(\mu^{-n}\right)$, because $\|g\|_{1}<\infty$ and $F$ is bounded. So to obtain an asymptotic expansion of $F(0, \mu)$ it is sufficient to obtain an asymptotic expansion of each integral $\mathbf{T}_{\mu}^{n}[\mathbf{1}](0)$.

We write

$$
\mathbf{T}_{\mu}[\mathbf{1}](0)=\int_{0}^{\infty} g(t) d t+\int_{0}^{\infty} e^{-\mu t} g(t) d t
$$

The first of these integrals is constant:

$c_{1}:=\int_{0}^{\infty}\left(\frac{5}{16} \frac{m^{2} x^{2 m-2}}{\left(x^{m}+1\right)^{3}}+\frac{m(m-1) x^{m-2}}{4\left(x^{m}+1\right)^{2}}\right) d \zeta=\frac{m}{32} B(2-1 / m, 1 / 2+1 / m)$,

where $d \zeta=\sqrt{Q(x)} d x$, and $B$ is Euler's Beta-function.

For the second integral in (36) we use the following lemma. Let us say that a function $h(x)$ expands into a convergent series of real powers if

$$
h(x)=\sum_{n=0}^{\infty} a_{n} x^{\beta_{n}}, \quad 0<x<\delta,
$$

where the exponents $\beta_{n} \rightarrow+\infty$ are real, and the convergence is absolute and uniform.

Lemma 1. Suppose that $h$ is integrable on the real line, and expands into a convergent series of real powers as in (37). Then

$$
\int_{0}^{\infty} e^{-\mu t} h(t) d t
$$

has an asymptotic expansion

$$
\sum_{n=0}^{\infty} a_{n} \Gamma\left(\beta_{n}+1\right) \mu^{-\beta_{n}-1}
$$

Proof of Lemma 1.

$$
\int_{0}^{\infty} e^{-\mu t} h(t) d t=\int_{0}^{\delta} e^{-\mu t} h(t) d t+O\left(e^{-\delta \mu}\right)=\sum_{n=0}^{\infty} a_{n} \int_{0}^{\delta} e^{-\mu t} t^{\beta_{n}} d t+O\left(e^{-\delta \mu}\right)
$$

and

$$
\int_{0}^{\delta} e^{-\mu t} t^{\beta_{n}} d t=\int_{0}^{\infty} e^{-\mu t} t^{\beta_{n}} d t+O\left(e^{-\delta \mu}\right)=\mu^{-\beta_{n}-1} \Gamma\left(\beta_{n}+1\right)+O\left(e^{-\delta \mu}\right) .
$$


This proves Lemma 1.

Applying Lemma 1 to the second integral in (36) we first expand $g$ in (25) and obtain

$$
g(t)=\frac{1}{4} m(m-1) t^{m-2}+O\left(t^{m-1}\right) .
$$

Then Lemma 1 implies that

$$
\int_{0}^{\infty} e^{-\mu t} g(t) d t=\frac{1}{4} m(m-1) \Gamma(m-1) \mu^{1-m}+O\left(\mu^{-m}\right),
$$

and this gives

$$
F(0, \mu)=1+c_{1} \mu^{-1}+\frac{1}{8} \Gamma(m+1) \mu^{-m}+O\left(\mu^{-m-1}\right)+O\left(\mu^{-2}\right) .
$$

This proves (16) when $m \in(1,2)$.

When $m>2$ one needs to show that for $n \geq 2$ the largest non-integer power of $\mu$ in the expansion of $\mu^{-n} \mathbf{T}_{\mu}^{n}[\mathbf{1}](0)$ is less than $-m$. To do this, we make the change of the variable in (35):

$$
x_{1}=t, \quad x_{2}=t_{1}-t, \quad x_{3}=t_{2}-t_{1}, \quad \ldots \quad x_{n}=t_{n-1}-t_{n-2},
$$

and break the integral (35) into the sum of $2^{n}$ integrals of the form

$$
I_{n, J}(\mu)=\int_{0}^{\infty} \ldots \int_{0}^{\infty} e^{-\mu L(\mathbf{x})} g\left(x_{1}\right) \ldots g\left(x_{1}+\ldots+x_{n}\right) d x_{n} \ldots d x_{1}
$$

where $L(\mathbf{x})=\sum_{j \in J} x_{j}$, and $J$ is a subset of $\{1, \ldots, n\}$. Then we have the following

Lemma 2. If $g$ is an analytic function in the sector $|\arg z|<\epsilon$ satisfying

$$
g(z)=O\left(z^{\alpha}\right), \quad z \rightarrow 0, \quad \text { and } \quad g(z)=O\left(z^{-2}\right), \quad z \rightarrow \infty
$$

where $\alpha>0$, then

$$
\mu^{-n} I_{n, J}=\sum_{k=0}^{[\alpha]+n+1} c_{k} \mu^{-k}+O\left(\mu^{-\alpha-1-n}\right), \quad \mu \rightarrow \infty .
$$

Our function $g$ satisfies conditions (39) with $\alpha=m-2$, so the contribution from $\mu^{-n} \mathbf{T}_{\mu}^{n}[\mathbf{1}](0)$ to (16) consists of integer powers and powers less than $-m$ of $\mu$, which will prove (16). 
Proof of Lemma 2. (F. Nazarov, private communication). Notice that derivatives $g^{(k)}$ are bounded on $[0, \infty)$ for $k \leq[\alpha]$. Moreover, all these derivatives satisfy

$$
g^{(k)}(z)=O\left(z^{-2}\right), \quad z \rightarrow \infty
$$

because $g$ is analytic in a sector and we can apply Cauchy's estimate to them. Put

$$
q=[\alpha]-1
$$

and write the Taylor formula with remainder for $g$ :

$$
g(u+v)=g(u)+g^{\prime}(u) v+\ldots+g^{(q)}(u) \frac{v^{q}}{q !}+\epsilon(u, v)
$$

where

$$
|\epsilon(u, v)| \leq M v^{q+1}
$$

This formula (42), (43) holds for all positive $u, v$, because $g^{(q+1)}$ is bounded on the whole positive ray.

For each $g\left(x_{1}+\ldots+x_{k}\right)$ in our integral, we split the argument into two summands:

$$
u_{k}=\sum_{j \notin J, j \leq k} x_{j} \text { and } v_{k}=\sum_{j \in J, j \leq k} x_{j},
$$

and apply (42) to $g\left(v_{k}+u_{k}\right)$. Notice that for each $k$

$$
v_{k} \leq L(\mathbf{x})
$$

Now we multiply these expressions obtained from the Taylor formula applied to each $g\left(x_{1}+\ldots+x_{k}\right)$, and split the integral into summands.

There are two types of summands.

Type 1. Those which do not contain any error term $\epsilon$. They are of the form

$$
\int_{0}^{\infty} \ldots \int_{0}^{\infty} e^{-\mu L(\mathbf{x})} g^{\left(j_{1}\right)}\left(u_{1}\right) \ldots g^{\left(j_{n}\right)}\left(u_{n}\right) v_{1}^{j_{1}} \ldots v_{n}^{j_{n}} d \mathbf{x}
$$

Notice that each variable $x_{j}$ participates either in some $u_{k}$ or in the exponential factor. This is because in $g\left(x_{1}+\ldots+x_{n}\right)$ all variables participate, and some term from the Taylor expansion of this function will enter each summand. So the integral is convergent in view of (26). Integration in the variables that are not in $J$ gives a constant factor, and the integration in 
the variables which are in $J$ gives integer power of $\mu$, because $v_{j}$ are honest polynomials (with integer powers of these variables).

Type 2. Those terms which contain at least one $\epsilon$. These terms are estimated using (43) and (44). All previous arguments are applicable to the estimates, (the estimates contain only integer powers), and these terms contribute to the asymptotics at most

$$
\mu^{-q-2}<\mu^{-\alpha-1}
$$

by Lemma $1^{1}$ and (41). Finally, the whole integral is divided by $\mu^{n}$ and we get that the top non-integer power in $I_{n, J}$ is at most

$$
\mu^{-\alpha-1-n}
$$

This proves Lemma 2 .

Analytic continuation and extension of the asymptotics to complex $\mu$.

First we keep $\mu$ positive and perform an analytic continuation of $\zeta \mapsto$ $F(\zeta, \mu)$ to the sector $|\arg \zeta|<\pi / m$. To do this we choose the path of integration in (30) to consist of two segments $\left[\zeta, \zeta_{0}\right]$ and $\left[\zeta_{0},+\infty\right)$, where $\zeta_{0}>\operatorname{Re} \zeta$. This defines our function $F(\zeta, \mu)$ in the sector, and $F(\zeta, \mu) \rightarrow 1$ as $\zeta \rightarrow \infty$ in this sector. Next we notice that the contour of integration can be changed to a ray $\left\{\zeta+r e^{i \phi}: r \geq 0\right\}$, as long as $|\phi|<\min \{\pi / m, \pi / 2\}$, without affecting the equation. This follows from Cauchy's theorem.

Once a ray of integration in (30) is fixed, the arguments in the previous part remain valid as long as $\left|e^{-\mu \zeta}\right|$ is decreasing on the ray of integration and tends to zero exponentially as $\zeta \rightarrow \infty$. For example, if the integration is along the positive ray, the asymptotics is valid for $|\arg \mu|<\pi / 2$. By rotating the integration ray, as explained above, we obtain the asymptotic expansion

$$
F(0, \mu)=1+\sum_{n=1}^{[m]} c_{n} \mu^{n}+(1 / 8) \Gamma(m+1) \mu^{m}+O\left(\mu^{-m-\epsilon}\right)
$$

which is valid in the sector $|\arg \mu|<\pi / 2+\pi / m-\epsilon$, for any $\epsilon>0$.

\footnotetext{
${ }^{1}$ We don't really need Lemma 1 here, but the trivial fact that if $P(x)$ is a monomial of degree $d$ in the variables participating in $J$, then

$$
\int \ldots \int e^{-\mu L(\mathbf{x})} P(\mathbf{x}) d \mathbf{x}=c \mu^{-d-1} .
$$
}


Recalling that $\lambda=\mu^{1 / \rho}$ we conclude that the required asymptotic expansion (16) holds in the sector $|\arg \lambda|<\pi-\epsilon$.

This completes the proof of Theorem 1 .

\section{Problem of level 1. Proof of Theorem 1.}

We consider the equation

$$
y^{\prime \prime}=\left(z^{m}+\lambda\right) y
$$

with $1<m<2$ and the boundary conditions

$$
y\left(r e^{ \pm 2 \pi i /(m+2)}\right) \rightarrow 0, \quad r>0, \quad r \rightarrow \infty .
$$

This is obtained by the change of the variable $z=i x$ in (2), and we have $\lambda=-E, m=2+\varepsilon$. The problem does not change when we rotate the normalization rays within their Stokes sectors, and $m \in(1,2)$, so the boundary condition (46) is equivalent to $y( \pm i r) \rightarrow 0$ as $r>0, r \rightarrow \infty$.

If $y_{0}(z, \lambda)$ is a solution of (45) then

$$
y_{k}(z, \lambda)=\omega^{k / 2} y_{0}\left(\omega^{-k} z, \omega^{2 k} \lambda\right),
$$

where

$$
\omega=e^{2 \pi i /(m+2)}, \quad \omega^{k / 2}=e^{\pi i k /(m+2)},
$$

is also a solution. We take as $y_{0}$ the solution normalized by (6).

Problem (45), (46) has discrete spectrum, and an entire function $C(\lambda)$ with zeros at the eigenvalues, which is called the spectral determinant, can be found from the following equation:

$$
C y_{0}=y_{1}+y_{-1} .
$$

See, for example, [8], [11, [16], [17]. Substituting $z=0$ we obtain

$$
C(\lambda)=\frac{\omega^{1 / 2} f\left(\omega^{2} \lambda\right)+\omega^{-1 / 2} f\left(\omega^{-2} \lambda\right)}{f(\lambda)} .
$$

It is a very restrictive property of an entire function $f$, that the zeros of denominator of (47) are canceled by the zeros of the numerator. In fact this property characterizes our $f$ under mild additional conditions, see the discussion in [11]. 


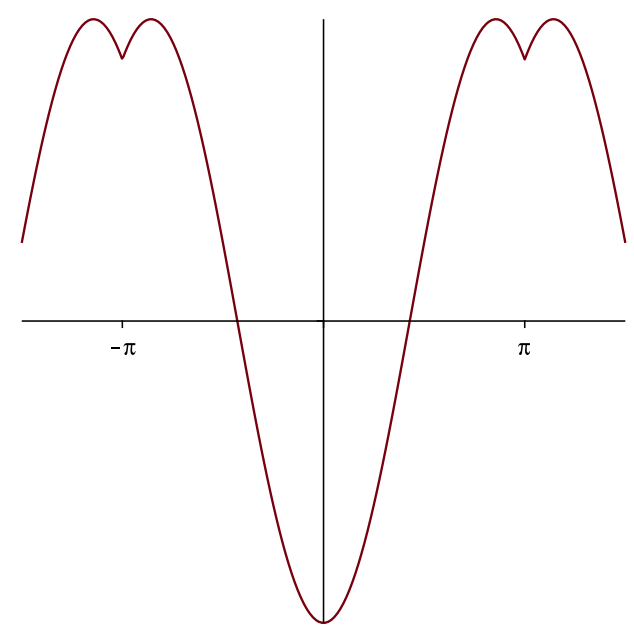

Figure 5: Indicator of $f$ when $m \in(1,2)$. The jump of the slope occurs at the accumulation point of the arguments of the zeros. Here $m=1.5$.

To investigate the zeros of $C$ we study the asymptotics of the numerator. The sum in this numerator can be zero only near the rays where asymptotics of the summands have equal absolute values. It is useful to consider the indicators of our entire functions [15]. In general, if $w$ is an entire function of order $\rho$, normal type, the indicator is defined by

$$
h_{w}(\theta)=\limsup _{r \rightarrow \infty} r^{-\rho} \log \left|w\left(r e^{i \theta}\right)\right|,
$$

so it shows the rate of growth of the function on the rays $\arg \lambda=\theta$. For all our functions the limit in the definition of the indicator exists for all rays except finitely many. It follows from (15), (16) that

$$
h_{f}(\theta)=K_{m} \cos \rho \theta, \quad|\theta| \leq \pi
$$

This indicator is shown in Fig. 5. We recall that $K_{m}<0$ for $m \in(1,2)$, the case considered in this section. Indicators are always $2 \pi$-periodic and continuous, and obey the following relations:

$$
\begin{gathered}
h_{w_{1} w_{2}}=h_{w_{1}} h_{w_{2}}, \\
h_{w_{1}+w_{2}} \leq \max \left\{h_{w_{1}}, h_{w_{2}}\right\},
\end{gathered}
$$




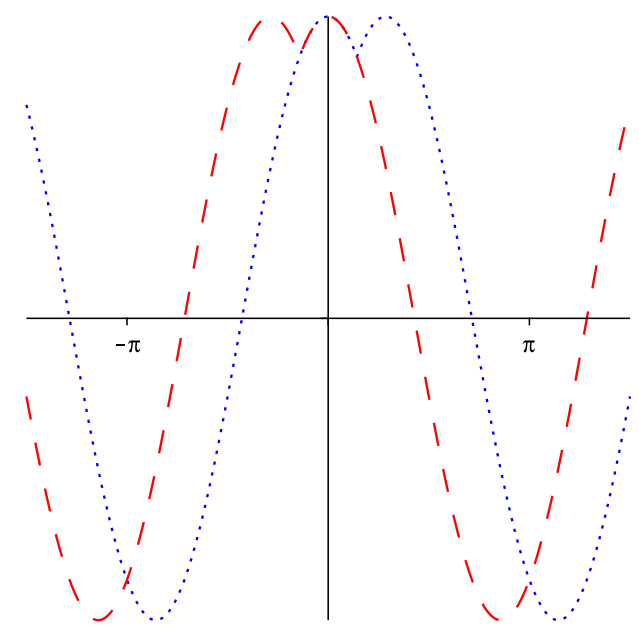

Figure 6: Indicators of $\omega^{1 / 2} f\left(\omega^{2} \lambda\right)$ and $\omega^{-1 / 2} f\left(\omega^{-2} \lambda\right)$.

and equality holds in the last inequality for each $\theta$ for which $h_{w_{1}}(\theta) \neq h_{w_{2}}(\theta)$. In our simple case, all indicators are piecewise trigonometric: they are solutions of the equation $h^{\prime \prime}+\rho^{2} h=0$ at every point except finitely many. The finitely many points where $h^{\prime}$ is discontinuous are the accumulation points of the arguments of zeros.

Indicators of the summands in the numerator of (47) are shown in Fig. 6. The indicator of the sum will be equal to the maximum of the indicators of the summands, if we show that there is no cancellation on the interval

$$
-\pi \frac{2-m}{2+m}<\theta<\pi \frac{2-m}{2+m}
$$

where the indicators of the summands are equal. To do this we use the asymptotics (15), (16).

So assume that

$$
|\arg \lambda|<\pi \frac{2-m}{2+m}
$$

and recall that $\rho$ is defined by (77). In computation with formula (16) one has to take into account that the principal branch is used for the powers of $\lambda$, so we have to replace every argument by a value on $(-\pi, \pi)$. Thus, for example

$$
\arg \left(\omega^{2} \lambda\right)=\frac{4 \pi}{m+2}+\arg \lambda-2 \pi=-\frac{2 \pi m}{m+2}+\arg \lambda \in(-\pi, \pi) .
$$


Now we have using (49):

$$
\omega^{1 / 2}\left(\omega^{2} \lambda\right)^{-1 / 4}=i \lambda^{-1 / 4}
$$

and

$$
\omega^{-1 / 2}\left(\omega^{-2} \lambda\right)^{-1 / 4}=-i \lambda^{-1 / 4} .
$$

Similarly

$$
\left(\omega^{2} \lambda\right)^{\rho}=\left(\omega^{-2} \lambda\right)^{\rho}=-\lambda^{\rho},
$$

and more generally, for integer $k$ :

$$
\left(\omega^{2} \lambda\right)^{-k \rho}=\left(\omega^{-2} \lambda\right)^{-k \rho}=(-1)^{k} \lambda^{-k \rho},
$$

In particular, this shows that all terms $\lambda^{k \rho}$ with integer $k$ cancel in the asymptotics of the sum $\omega^{1 / 2} f\left(\omega^{2} \lambda\right)+\omega^{-1 / 2} f\left(\omega^{-2} \lambda\right)$. However

$$
\left(\omega^{ \pm 2} \lambda\right)^{-m \rho}=\omega^{\mp 2 m \rho} \lambda^{-m \rho}=e^{ \pm i \pi m} \lambda^{-m \rho},
$$

where we used (49). Combining all these calculations, we conclude that

$$
\omega^{1 / 2} f\left(\omega^{2} \lambda\right)+\omega^{-1 / 2} f\left(\omega^{-2} \lambda\right)=-2 \exp \left(-K_{m} \lambda^{\rho}\right)\left(\lambda^{-m \rho} \sin \pi m+o\left(\lambda^{-m \rho}\right)\right),
$$

when $\lambda$ satisfies (48). This shows that when $m$ is not an integer, there is no complete cancellation of the asymptotic expansions, and the indicator of the numerator in (47) is the maximum of the indicators of the summands, and also shows that the spectral determinant $C(\lambda)$ has no zeros in the sector (48). All this is true when $m \in(1,2)$.

Remark 1. To see what is the difference between the cases $m<2$ and $m>2$, we sketch the indicators of the numerator in this case in Fig. 7; notice that for $m>2$, the indicator of $f$ looks like Fig. 8 , because $K_{m}>0$. Fig. 7 shows that there are zeros of the numerator of (47) whose arguments accumulate to 0 , and in fact there are infinitely many positive zeros as proved in [11].

Now we return to the proof of Theorem 1. Indicator of the numerator in (47) in Fig. 6 shows that the only possible direction of accumulation of the arguments of zeros of $C$ can be the negative ray $\arg \lambda=\pi$, and the rays $\arg \lambda= \pm \pi(2-m) /(m+2)$. Indeed there are infinitely many zeros of the numerator with arguments accumulating to these three points. We will show 


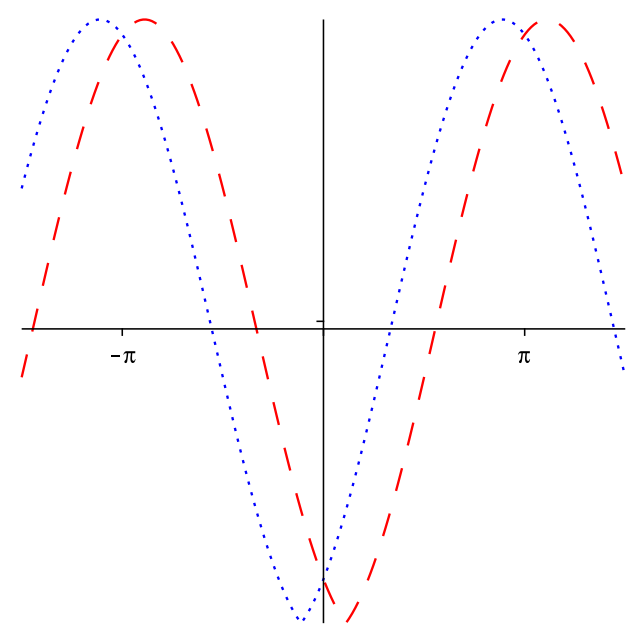

Figure 7: Indicators of $\omega^{1 / 2} f\left(\omega^{2} \lambda\right)$ and $\omega^{-1 / 2} f\left(\omega^{-2} \lambda\right)$ for $m>2$. Here $m=$ 2.5 .

that zeros with arguments near $\pi$ are actually real, and almost all of them cancel with the zeros of the denominator $f$.

To do this we first show that the asymptotics of the numerator and denominator of (47) differ by an exponential factor. Indeed, for

$$
\pi-\epsilon<\arg z<\pi+\epsilon
$$

where $\epsilon$ is sufficiently small, we have in view of (13)

$f(\lambda)=\lambda^{-1 / 4} \exp \left(K_{m} \lambda^{\rho}+O\left(\lambda^{-\rho}\right)\right)+i \lambda^{-1 / 4} \exp \left(-K_{m} e^{-2 \pi i / m} \lambda^{\rho}+O\left(\lambda^{-\rho}\right)\right)$,

where we used the fact that $e^{-2 \pi i \rho}=-e^{-2 \pi i / m}$. On the other hand, in the same sector (50) we have

$$
\begin{aligned}
\omega^{1 / 2} f\left(\omega^{2} \lambda\right)+\omega^{-1 / 2} f\left(\omega^{-2} \lambda\right) & =i \lambda^{-1 / 4} \exp \left(-K_{m} \lambda^{\rho}+O\left(\lambda^{-\rho}\right)\right) \\
& +\lambda^{-1 / 4} \exp \left(K_{m} e^{-2 \pi i / m} \lambda^{\rho}+O\left(\lambda^{-\rho}\right)\right),
\end{aligned}
$$

and multiplying this by the exponential factor

$$
\exp \left(\left(\left(1-e^{-2 \pi i / m}\right) K_{m} \lambda^{\rho}\right)\right.
$$

we obtain (51). 
Now we follow the arguments from [17]. Let $\lambda$ be a root of $f$. We conclude from (13), (14) (which is the same as (151)) that

$$
\exp \left(K_{m} \lambda^{\rho}+O\left(\lambda^{-\rho}\right)\right)=-i \exp \left(K_{m} e^{-2 \pi i \rho}+O\left(\lambda^{\rho}\right)\right) .
$$

Denoting $\lambda^{\rho}=e^{\pi i \rho} \zeta$, we obtain

$$
\zeta_{n}=a n+b+O\left(n^{-1}\right)
$$

where

$$
a=\frac{\pi}{K_{m} \sin \pi \rho}, \quad b=-\frac{\pi}{4 K_{m} \sin \pi \rho} .
$$

This implies the approximate formula for the roots of (52)

$$
-\lambda_{n}=(a n)^{1 / \rho}+(b / \rho)(a n)^{1 / \rho-1}+O\left(n^{1 / \rho-2}\right) .
$$

As the asymptotics of $f$ and of the numerator of (47) differ only by an exponential multiple, we conclude that the same formula (53), with the same $a$ and $b$, also holds for the roots of the numerator of (47) near the negative ray. The error term $O\left(n^{1 / \rho-2}\right)$ in this formula tends to zero faster than the distance between the neighboring roots. Indeed this distance is at least

$$
c_{1}\left((n+1)^{1 / \rho}-n^{1 / \rho}\right)>c_{2} n^{1 / \rho-1},
$$

where $c_{1}$ and $c_{2}$ are some positive constants. As $C$ is entire, each root of $f$ must cancel with some root of the numerator of (47), and we conclude that almost all roots of this numerator near the negative ray cancel. Therefore, $C$ has finitely many roots near the negative ray.

This completes the proof of Theorem 1.

\section{Problem of level 2. Proof of Theorem 2.}

The spectral determinant of the level 2 problem is

$$
D(\lambda)=\frac{\omega f(\omega \lambda) f\left(\omega^{3} \lambda\right)+\omega^{-1} f\left(\omega^{-1} \lambda\right) f\left(\omega^{-3} \lambda\right)+f\left(\omega^{3} \lambda\right) f\left(\omega^{-3} \lambda\right)}{f(\omega \lambda) f\left(\omega^{-1} \lambda\right)},
$$

see, for example [1]. This is obtained from the formula

$$
D(\lambda)=C(\omega \lambda) C\left(\omega^{-1} \lambda\right)-1,
$$

which is derived in [8], [11] and [16], by substituting the expression (47) for $C$. The order $\rho$ of $f$ is less than 1 now, and the indicator of $f$ is shown in 


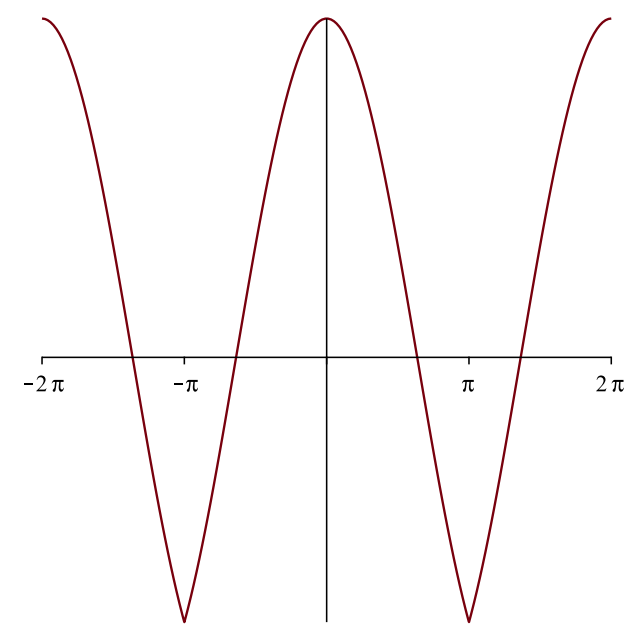

Figure 8: Indicator of $f$ for $m>2$. Here $m=3.5$.

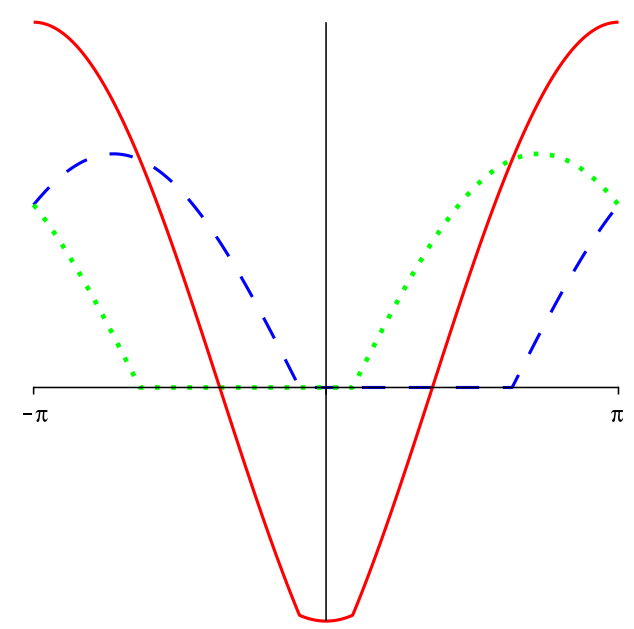

Figure 9: Indicators of the three summands in the numerator of (154). 
Fig. 8. The indicators of the three summands in the numerator on $D$ are shown in Fig. 9.

We have to verify that there is no cancellation, so that the indicator of the numerator in (54) is indeed zero on the interval around 0 . We compute the asymptotic expansion of this numerator, neglecting the third summand $f\left(\omega^{3} \lambda\right) f\left(\omega^{-3} \lambda\right)$ whose indicator is negative on the interval under consideration.

Beginning with $\omega f(\omega \lambda) f\left(\omega^{3} \lambda\right)$, we use (16), and restrict $\lambda$ to the sector

$$
|\arg \lambda|<\pi \frac{4-m}{2+m} .
$$

For such $\lambda$, we have the following principal values of the arguments:

$$
\begin{gathered}
\arg \omega \lambda=\frac{2 \pi}{m+2}+\arg \lambda, \\
\arg \omega^{3} \lambda=\frac{6 \pi}{m+2}-2 \pi+\arg \lambda=-\frac{2 \pi(m-1)}{m+2}+\arg \lambda \in(-\pi, \pi) .
\end{gathered}
$$

Using (7), we conclude that

$$
\omega^{\rho}+\left(\omega^{3}\right)^{\rho}=0
$$

therefore the exponential term in $\omega f(\omega \lambda) f\left(\omega^{3} \lambda\right)$ disappears, as seen in Fig. 9. The multiple coming from $\lambda^{-1 / 4}$ is

$$
\omega(\omega \lambda)^{-1 / 4}\left(\omega^{3} \lambda\right)^{-1 / 4}=i \lambda^{-1 / 2}
$$

and similarly

$$
\omega^{-1}\left(\omega^{-1} \lambda\right)^{-1 / 4}\left(\omega^{-3} \lambda\right)^{-1 / 4}=-i \lambda^{-1 / 2} .
$$

The rest is an asymptotic series in negative powers of $\lambda^{\rho}$. We claim that the top non-integer power of $\lambda^{\rho}$ in this series for the numerator of (54) does not vanish. Indeed, this term is $\Gamma(m+1) / 8$ multiplied by

$$
\begin{aligned}
& i\left((\omega \lambda)^{-m \rho}+\left(\omega^{3} \lambda\right)^{-m \rho}\right)-i\left(\left(\omega^{-1} \lambda\right)^{-m \rho}+\left(\omega^{-3} \lambda\right)^{-m \rho}\right) \\
= & i\left(\left(e^{i \pi m}-e^{-i \pi m}\right) \lambda^{-m \rho}=2 \lambda^{-m \rho} \sin \pi m \neq 0,\right.
\end{aligned}
$$

when $m$ is not an integer. 
Thus $D$ has infinitely many zeros with arguments accumulating to the boundary rays of the sector (55). The numerator also has zeros with arguments accumulating to $\pm 2 \pi /(m+2)$ but almost all these zeros cancel with the zeros of the numerator. This is proved by a reasoning similar to that in the previous section. This completes the proof of our theorem.

Remark 2. When $m$ is an integer, the asymptotic expansion in (16) contains only integer powers, and we must have complete cancellation of all terms of the asymptotic expansion of the numerator of (54).

Remark 3. It is very plausible that the pattern proved in this paper persists for all levels, and the pictures of indicators of the spectral determinants suggest that the same proof can be extended.

The authors thank Victor Katsnelson, Fedja Nazarov and Kwang Shin for useful discussions.

\section{References}

[1] C. Bender and S. Boettcher, Real spectra in non-Hermitian Hamiltonians having PT symmetry. Phys. Rev. Lett. 80 (1998), no. 24, 5243-5246.

[2] C. Bender and S. Boettcher, PT-symmetric quantum mechanics, J. Math. Phys. 40 (1999), no. 5, 2201-2229.

[3] C. Bender, N. Hassanpour, D. Hook, S. Klevansky, C. Sünderhauf, and Zichao Wen, Behavior of eigenvalues in a region with broken PT symmetry, Phys. Rev. A 95 (2017) 052113.

[4] C. Bender and Tai Tsun Wu, Tsun Anharmonic oscillator, Phys. Rev. (2) 1841969 1231-1260.

[5] M. Born and P. Jordan, Zur Quantummechenik, Z. Phys., 34 (1925) p. 858. English transl.: V. L. van der Waerden, Sources of quantum mechanics, North Holland, Amsterdam, 1967, 277-306.

[6] N. Bourbaki, Fonctions d'une variable réelle, Herman, Paris, 1951.

[7] P. Dorey, C. Dunning and R. Tateo, Spectral equivalences, Bethe ansatz equations, and reality properties in PT-symmetric quantum mechanics. J. Phys. A 34 (2001), no. 28, 5679-5704. 
[8] P. Dorey, C. Dunning and R. Tateo, The ODE/IM correspondence. J. Phys. A 40 (2007), no. 32, R205-R283.

[9] P. Dorey, C. Dunning and R. Tateo, From PT-symmetric quantum mechanics to conformal field theory, Pramana - journal of physics, 73 (2009) 217-239.

[10] P. Dorey, A. Millican-Slater and R. Tateo, Beyond the WKB approximation in PT-symmetric quantum mechanics, J. Phys. A 38 (2005), no. $6,1305-1331$.

[11] A. Eremenko, Entire functions, PT-symmetry and Voros's quantization scheme, arXiv:1510.02504.

[12] J. Heading, Introduction to phase-integral methods, John Willey \& Sons, Inc., NY 1962.

[13] M. V. Fedoryuk, Asymptotics. Integrals and series (Russian) Moscow, Nauka, 1987.

[14] W. Heisenberg, Über quantentheoretische Umdeutung kinematischer und mechanischer Bezeichingen, Z. Phys., 33 (1925) p. 839. English transl.: V. L. van der Waerden, Sources of quantum mechanics, North Holland, Amsterdam, 1967, 261-276.

[15] B. Ya Levin, Distribution of zeros of entire functions, AMS, Providence RI, 1980.

[16] K. Shin, The potential $(i z)^{m}$ generates real eigenvalues only under symmetric rapid decay conditions, J. Math. Phys., 46 (2005) 082110.

[17] K. Shin, Asymptotics of eigenvalues of non-self-adjoint Schrödinger operators on a half-line, Comp. methods and function theory, 10 (2010) 2, $111-133$.

[18] Y. Sibuya, Global theory of a second order linear ordinary differential equation with a polynomial coefficient, North-Holland, NY, 1975.

[19] T. Tabara, Asymptotic behavior of Stokes multipliers for $y^{\prime \prime}-(x \sigma+\lambda) y=$ $0(\sigma \geq 2)$ as $\lambda \rightarrow \infty$, Differential equations and dynamical systems (Waterloo, ON, 1997). Dynam. Contin. Discrete Impuls. Systems 5 (1999), no. 1-4, 93-105. 
[20] A. Voros, The return of the quartic oscillator: the complex WKB method, Ann. Inst. H. Poincaré Sect. A, 39 (1983), no. 3, 211-338.

Department of Mathematics

Purdue University

West Lafayette, IN 47907

USA

eremenko@math.purdue.edu

agabriel@math.purdue.edu 